\title{
Use of remote sensing in zoning's studies for terroir and precision viticulture: Implementation in DO Ca Rioja (Spain) I Uso de la teledetección en los estudios del terroir para la viticultura de precisión: Aplicación en la DO Ca Rioja (España)
}

\author{
Vicente D. Gómez-Miguel ${ }^{1, a}$, Vicente Sotés ${ }^{1}$, Álvaro Martínez ${ }^{1}$, and y María L. González-SanJosé2 \\ ${ }^{1}$ Departamento de Producción Agraria, Universidad Politécnica de Madrid (UPM), España \\ ${ }^{2}$ Department of Biotechnology and Food, Science, Universidad de Burgos (UBU), España, \\ Contacto: C/ Puerta de Hierro, 2, 28040 Madrid
}

\begin{abstract}
Resumen. Existen dos formas principales de gestión de la parcela/explotación: el Manejo Tradicional o Convencional (MC) y la Viticultura de Precisión (VP). Aunque las exigencias tecnológicas de ambos son diferentes, tanto en uno como en otra, es necesario el Mapa de Zonificación del Terroir o Mapa de Variabilidad del Terroir (MVT). El MVT constituye la cartografía detallada de la parcela y permite conocer la delimitación y la caracterización de los diferentes sectores que constituyen su variabilidad. La VP gestiona de forma independiente y optimizada cada uno de estos sectores, permitiendo, entre otras muchas cosas, la optimización del diseño de la plantación, la selección de patrones, el laboreo, el manejo de cubiertas, la adecuación de la fertilización de fondo y mantenimiento, el ajuste de la nutrición hídrica, el diagnóstico y tratamiento de plagas y enfermedades, el seguimiento de la maduración, la elección del momento de la vendimia, etc. y todo ello de manera localizada en función las características propias de cada sector. El Método Directo (MD) es la forma usual de conseguir el MVT. El mapa realizado a partir del Método Directo delimita y caracteriza los diferentes sectores con eficacia y además aporta información sobre las propiedades del terroir, lo que permite diagnosticar las causas de la heterogeneidad y con ello la posibilidad de manejarlas adecuadamente. Los mapas obtenidos por sensores remotos (teledetección) a partir de Imágenes Espectrales de Alta Resolución (IEAR) aportan información importante, son muy asequibles, facilitan la precisión de los límites y se obtienen más rápidamente que los realizados a partir del MD. Sin embargo, este tipo de mapas separa zonas cuya diferenciación se debe a la respuesta de la planta a una determinada situación que puede estar originada por un sinfín de posibilidades y, por lo tanto varían con ellas. Estos mapas son incapaces de discriminar sectores por las propiedades de los factores del medio (clima, litología, geomorfología, suelo) que afectan a la variabilidad del terroir lo que imposibilita la actuación directa sobre ellas. La ejecución del MVT realizado a partir del MD se encarece a grandes escalas (estudios muy detallados) debido a la dificultad de realizar adecuadamente las delineaciones (el error con malla fija es función de la longitud de los lados). Sin embargo, la tecnología del mapa obtenido de IEAR permite amortiguar este problema y disminuir el coste. Tanto en los mapas obtenidos por el MD como los obtenidos de IEAR la calidad del muestreo es fundamental. Tanto la densidad de observaciones como el número de propiedades implicadas en el estudio determinan su utilidad y las directrices, a ser posible siguiendo normas internacionales (como por ejemplo, OIV), deben ser garantía de calidad. En definitiva, ambas metodologías, MD e IEAR, se complementan para la realización del MVT y, una vez que se dispone del mapa realizado a partir del MD, son posibles las interpretaciones correctas de los mapas obtenidos mediante IEAR, ya sean anuales, estacionales, en función del estado fenológico o por cualquier otra circunstancia que pueda afectar a la variabilidad (plagas, estrés,...). El MVT así obtenido permite al viticultor abordar el objetivo más ambicioso: la optimización y homogeneización del producto. En concreto se trata de que a corto o medio plazo (futuro) y mediante la gestión llevada a cabo con la VP la actuación sobre las propiedades del terroir en los diferentes sectores de la parcela se consiga la mayor homogeneidad posible y así también el producto lo sea. En este trabajo tratamos de la variabilidad del terroir y de los sistemas de gestión del viñedo (MC y VP) destacando la importancia de la cartografía y la necesidad de los MVT y su realización complementando el MD y la utilización de los sistemas de percepción remota (IEAR) como otro método de diferenciación.
\end{abstract}

\begin{abstract}
There are two principal ways of plot/exploitation management: Traditional or Conventional Managing (MC) and Precision viticulture (VP). Even though the technological requirements from these systems are different, in both cases, the Zoning or Variability Map for the Terroir (MVT) is required. MVT is a detailed mapping of the plot and enables to know the delineation and characterization of several
\end{abstract}

\footnotetext{
a e-mail: Vicente.gomez@upm.es
}

(c) The Authors, published by EDP Sciences. This is an Open Access article distributed under the terms of the Creative Commons Attribution License 4.0 (http://creativecommons.org/licenses/by/4.0/). 
sectors which make up its variability. The VP manages independently and optimized each of these sectors, allowing, among other things, optimizing the plantation's layout, rootstocks' selection, tillage, cover crop management, fertilization and maintenance programs, adjustment of water needs, diagnosis and pests and diseases management, monitoring of ripening, the harvest timing, etc. and all so in a localized manner according to the characteristics of each sector. Direct Method (MD) is the natural way to get the MVT. The map made from Direct Method defines and characterizes the different sectors indeed, and also provides some information about the terroir characteristics, which allow diagnosing the causes of heterogeneity and thus, handle them properly. Maps obtained by remote sensing from Spectral High Resolution Imaging (IEAR) provide relevant information, they are very affordable, and with an easy accuracy limits and they are obtained quicker than those made from MD. However, this kind of maps split the areas whose differentiation is due to the plant response in a specific situation that may be caused by a nearly endless options, and therefore, they vary with them. These maps are unable to discriminate sectors by the properties according to environmental factors (climate, lithology, geomorphology, soil) that affect the terroir variability, forbidding any direct action on them. Doing a MVT performed through a MD is expensive on large scales (very detailed studies) due to the gap about adequate perform delineations (net fixed error is related to sides length). However, IEAR technology provides a map which is obtained allowing to buffer this problem and also, reducing the costs. Both in maps obtained from MD or IEAR, the sampling quality is a key point. Both density of observations and the number of properties involved in the study determine its utility and the relative directives, when is possible under an international procedure (such as, OIV), must be a quality guarantee. In fact, both methodologies (MD and IEAR) are complementary for doing the MVT and, once the map (done through MD) is available, the right interpretations of the IEAR maps are possible, whether annual, seasonal, depending on the phenological status or any other circumstance that may affect the variability (pests, stress, ...). MVT obtained like that, allows to the viticulturist address the most ambitious goal: the product optimization and homogenization. Specifically, in a short or medium term and by the management carried out with the VP's performance on the terroir's properties in different sectors of the parcel/plot, resulting in the highest uniformity as possible, and also, in the product. In this work, we worked on terroir's variability and the vineyard management systems (MC and VP), highlighting the relevance of mapping, the need for MVT and its complementary performance related to the MD, and the use of remote sensing systems (IEAR) as another discriminative method.

Résumé. Il y a deux manières dans la gestion de la parcelle : l'entretien traditionnel ou conventionnel (MC) et la viticulture de précision (VP). Bien que des exigences technologiques parmi eux soient différentes, dans les deux cases, la carte du zonage ou de la variabilité du terroir (MVT) est toujours nécessaire. Le MVT constitue la cartographie détaillée du terrain et révèle la délimitation et la caractérisation des différents secteurs qui composent sa variabilité. Le VP géré de façon indépendante et optimisé chacun de ces secteurs, ce qui permet, parmi d'autres, l'optimisation de la mise en page de la plantation, sélection de porte-greffes, le travail du sol, la gestion des couvertures végétales, première fertilisation du sol et son entretien, l'optimisation de l'eau, le diagnostic et le traitement des maladies et ravageurs, la motorisation de la maturation, le moment de récolte, etc., et tout bien localisée selon des caractéristiques de chaque secteur. Le Méthode Direct (MD) est la manière plus utilisée pour réussir au MVT. La carte qui se réalise à partir du Méthode Direct, définit et caractérise les différents secteurs efficacement et fournit également des informations sur les propriétés du terroir, qui permet diagnostiquer des causes de l'hétérogénéité et donc, la chance de les gérer correctement. Les cartes obtenues par télédétection sur la basse de imagerie spectrales à haute résolution (IEAR) fournissent des informations importantes et sont très abordables, ses limites de précision sont faciles et sont obtenus plus vite par rapport à ceux fabriqués selon des MD. Cependant, ce type de cartes sépare des zones dont la différenciation est due à la réponse de la plante selon une situation particulière qui peut être provoquée par une milliard de possibilités et alors, elle peut varier avec eux. Ces cartes sont incapables pour distinguer les secteurs par ses propriétés selon des facteurs environnementaux (climat, lithologie, géomorphologie, sol) qui affectent la variabilité du terroir et en excluent l'action directe sur eux. Le MVT fourni à partir du MD se rendre plus cher à grandes échelles (études très détaillées), selon la difficulté de l'effectuer adéquatement et ses délimitations (l'erreur de maillage fixe est fonction de la longueur des côtés). Cependant, la technologie IEAR donne une carte qui permet d'amortir ce problème et réduire son coût. Même dans des cartes obtenues par le MD comme ceux obtenus d'IEAR, la qualité de l'échantillonnage est fondamentale. Bien la densité des observations et le nombre de propriétés impliquées dans l'étude, déterminent leur utilité et les lignes directrices, si possible selon les normes internationales (telles que OIV) qui doivent donner une assurance de qualité. En fin, ce deux méthodologies (MD et IEAR) sont complémentaires pour bien faire le MVT, et une fois que la carte réalisé à partir du MD soit disponible, les interprétations correctes des cartes obtenues par IEAR seront possibles, bien annuelles, saisonnières, selon l'état phenologique ou pour d'autre circonstance qui peut affecter à la variabilité (plages, stress,...). Le MVT obtenu permet au viticulteur aborder le but le plus ambitieuse : l'optimisation et homogénéisation du produit. En clair, il s'agit de que au court ou moyen terme et selon la gestion réalisée avec la performance du VP sur les propriétés du terroir dans les différents secteurs de la parcelle, avoir réussi la plus uniformité qui soit possible et au même temps, la plus haute sur le produit. Dans ce travail, nous avons traité la variabilité des systèmes du terroir et de gestion du vignoble (MC et VP) soulignant l'importance de la cartographie, la nécessité des MVT et sa réalisation en complément le $\mathrm{MD}$ correspondent, et l'utilisation de systèmes de télédétection (IEAR) comme d'autre méthode de différenciation possible. 


\section{Introducción}

La calidad del vino está determinada por los diferentes elementos o propiedades de un conjunto de factores naturales, biológicos, agronómicos y enológicos que son o pueden ser modificados por el hombre mediante lo que se describe frecuentemente con los términos gestión o manejo. El conjunto de todos estos elementos es lo que constituye el terroir.

La gestión o manejo del viñedo "pretende utilizar todas las hectáreas, dentro de su capacidad y tratarlas de acuerdo a sus necesidades" (H. H. Bennett cit. USDA, 2007) e incluye decisiones sobre la elección del sitio, el diseño de la plantación, la elección del portainjerto y la variedad, la formación de la planta, el laboreo, la nutrición mineral, la alimentación hídrica, la elección y manejo de cubiertas, el control sanitario, la vendimia, etc. Existen dos formas principales de gestión o manejo: El Manejo tradicional o convencional (MT-MC) y la Viticultura de Precisión (VP).

En el caso del MC toda la parcela, entendida como unidad de gestión, es tratada de forma homogénea, de manera que las necesidades del sector limitante en cuanto a calidad, producción o cualquier otro objetivo, o bien condicionan el manejo (laboreo, aporte de fertilizante, dosis de riego, etc) del conjunto, o bien es ignorado en beneficio de otro considerado de más importancia por su extensión, productividad, calidad o cualquier otra circunstancia preferente.

La Viticultura de Precisión (VP) es una forma de gestión de la explotación que se basa en herramientas que ponen a disposición del viticultor información georreferenciada del viñedo, apoyándose principalmente en la separación cartográfica (intra)parcela de sectores (subzonas) diferenciados por propiedades susceptibles de influir tanto en la calidad del producto resultante como en el propio manejo del viñedo. Las variaciones locales de los factores del clima, de la litología, de la geomorfología y del suelo afectan al desarrollo de la vid, a la composición de la uva y a la calidad del vino y por ello determinan la existencia de los sectores que definen las unidades que condicionan o limitan la VP (Gómez-Miguel 2011).

Anteriormente a la aparición de la VP, la variabilidad dentro de una parcela se manejaba en gran medida como ruido y con demasiada frecuencia no se apreciaba, y por lo tanto no se podía explicar y mucho menos relacionar y gestionar el conjunto de los parámetros implicados en esta variabilidad (Cook y Bramley, 1998; Bramley y Hamilton, 2004, 2007).

En general, hay muchas definiciones que se refieren a la Agricultura de Precisión y cada una de ellas está a menudo condicionada por el equipo comercial o la tecnología que actualmente constituya una novedad o simplemente esté de moda. De acuerdo con la propuesta de OIV (OIV, 2015) "la VP es un proceso cíclico continuo que incluye la recopilación de datos, el análisis de datos, la delimitación de zonas de manejo, las decisiones de gestión y la evaluación de las prácticas aplicadas".

La cartografía detallada de la parcela permite conocer la variabilidad entre tales sectores y la VP trata de forma independiente y optimizada cada uno de ellos, permitiendo, entre otras muchas cosas, la optimización del diseño de la plantación, la selección de portainjertos, la adecuación de la fertilización de fondo y mantenimiento, el ajuste de la nutrición hídrica, el diagnóstico y tratamiento de plagas y enfermedades, el seguimiento de la maduración, la elección del momento de la vendimia, etc. y todo ello de manera localizada, en función las características propias de cada sector.

La representación gráfica de los resultados en los diferentes sectores definidos por las características del medio se lleva a cabo a través de su cartografía, y la técnica de ejecución de los mapas se ha facilitado y mejorado sensiblemente con el uso de Sistemas de Información Geográfica (SIG o GIS). La realización de estos mapas, y especialmente del mapa de suelos, es un trabajo multidisciplinar, complejo y costoso, pero tales mapas son la base de la VP ya que permiten no sólo la caracterización de cada sector y la diferenciación entre sectores (subzonas), sino también explicar los efectos de esta variabilidad en el viñedo señalando sus verdaderas causas. Estas causas son importantes sobre todo porque actuando sobre ellas con un manejo adecuado se mejora el resultado, se homogeneiza el producto y se aumenta la calidad del vino.

Una de las formas de discriminar las diferencias existentes en el comportamiento entre plantas situadas dentro de un mismo viñedo (intraparcela) o en viñedos distintos (interparcela) es a través de sensores remotos. Desde finales del siglo pasado se han utilizado para este objetivo Índices de Vegetación (IV) muy diversos calculados a partir de imágenes multiespectrales.

En definitiva, el objetivo directo de la zonificación es la identificación de las causas de la variabilidad del terroir y la obtención del mapa de variabilidad del viñedo intraparcela para su utilización en VP como sistema de gestión o manejo de la explotación, En este trabajo se trata de utilizar los datos obtenidos a partir de determinados sensores de percepción remota (remote sensing) como herramienta para la optimización de las técnicas cartográficas tradicionales utilizadas en los estudios de macrozonificación.

En concreto, trataremos primero la variabilidad del terroir y de los sistemas de gestión del viñedo destacando la importancia de la cartografía y la necesidad de los mapas de variabilidad y su realización y posteriormente nos referiremos a las diferentes fuentes de información y a la utilización de los sistemas de percepción remota como otro método de diferenciación.

La variabilidad de una determinada situación puede ser debida a cualquier diferencia en cada uno de tales elementos o propiedades de cada uno de los factores que componen el terroir incluida la gestión o el manejo.

En definitiva, aunque las exigencias tecnológicas de las dos posibilidades de sistemas de gestión de la parcela/explotación son diferentes, existe una sola solución para realizarlas de forma conveniente: tanto en el MC/MT como en la VP es necesario el mapa de variabilidad del terroir como punto de partida para la correcta definición de las unidades de manejo.

\section{Mapas de variabilidad del terroir}

En general el viticultor no está bien informado de las propiedades del lugar en el que se desarrolla su viñedo y lo gestiona de forma convencional (es decir, uniforme, MC-MT) a partir del conocimiento que tiene sobre el rendimiento: la calidad apenas se considera o simplemente se ignora. Los rendimientos varían generalmente (hasta 
más de 10 veces) y sus patrones de variación son estables en el tiempo. La calidad del fruto también es variable, y sigue patrones de variación similares a los del rendimiento.

La variabilidad del medio que soporta la viña y las dificultades que esta conlleva para una gestión adecuada (variabilidad del terroir) están en el origen de la heterogeneidad del producto y en definitiva de la calidad del vino.

Esta variabilidad es siempre más fácil de detectar que de describir, caracterizar y cartografiar. Las dificultades para la caracterización y cartografía de las partes de la parcela que son similares y su separación de aquellas que son diferentes es lo que hace imprescindible un mapa al que nos referimos como de variabilidad o sus sinonimias:

Mapa de Variabilidad del terroir $\leftrightarrow$ Mapa de los terroir $\leftrightarrow$ Mapa de Zonificación del terroir

En definitiva este mapa permite la "caracterización espacial de zonas semejantes para producir uvas o vinos de composiciones similares, aun cuando permitan decisiones operacionales a distintos niveles de producción" (Vaudour, 2003).

La utilidad de este mapa es múltiple y sus potencialidades dependen de su calidad y de la información aportada por este. El uso de un enfoque técnico moderno para la evaluación y el reconocimiento del lugar y para el muestreo del propio viñedo produce una información importante que de otro modo puede perderse.

La calidad del mapa de variabilidad radica en la de las fuentes de información y en las técnicas cartográficas utilizadas para su realización, en la precisión y exactitud requerida por una escala adecuadamente elegida de acuerdo con los objetivos, en la densidad de observaciones y en las técnicas de muestreo del suelo y de la planta seleccionados y en la importancia y el número de propiedades, variables o parámetros considerados.

La escala del mapa debe estar de acuerdo con los objetivos del trabajo (Fig. 5): mientras que para un estudio de ordenación del cultivo de la vid en una determinada denominación de origen es necesaria una escala media (por ejemplo, 1:25.000), para un mapa de variabilidad de una explotación que pretenda aplicar técnicas de manejo relacionadas con la VP es conveniente una escala grande (por ejemplo, 1:5.000).

La densidad de observaciones relacionadas con el mapa de suelos está condicionada por la escala elegida $\mathrm{y}$, junto con el número de determinaciones que se han de realizar en el laboratorio de acuerdo con los objetivos previstos, determinan su coste.

El suelo se estudia a partir de su perfil y consta de una o varias capas llamadas horizontes. El conjunto de los horizontes A y B se denomina solum y constituye la profundidad efectiva en la que se desarrolla la mayor parte del sistema radicular de la planta, de ahí su importancia en la alimentación hídrica y nutricional de la propia planta. Esto es importante porque cada horizonte tiene sus propiedades y no es correcto referirse, por ejemplo, a un suelo arcilloso o arenoso sino a un horizonte arcilloso o arenoso de un suelo determinado; por ejemplo, en los viñedos no es infrecuente que el horizonte $\mathrm{A}$ sea franco arenoso, el B arcilloso y el C franco. Esto incrementa sensiblemente el número de muestras que se han de realizar y encarece el estudio.
Las propiedades que se deben caracterizar en el campo y las determinaciones que han de realizarse en el laboratorio a la muestra correspondiente de cada uno de los horizontes del perfil son las que definen la información disponible para el viticultor $\mathrm{y}$, por lo tanto, también establecen la calidad del estudio. El muestreo del viñedo está condicionado principalmente por el objetivo y por el tipo de análisis elegido.

\section{Realización del mapa de variabilidad}

La información para la realización del mapa de variabilidad de los terroir procede principalmente de la procedente del tratamiento de imágenes obtenidas mediante sensores remotos (Percepción remota) o de la obtenida por técnicas cartográficas que incluyen el muestreo de campo y los análisis de laboratorio y que son utilizadas por disciplinas como la Climatología, la Geología, la Geomorfología, la Edafología o la Botánica (Método directo).

De forma general se puede afirmar que la variabilidad del suelo es de onda muy corta y que por lo tanto es uno de los elementos del terroir que le aporta mayor variabilidad. La visibilidad en las fotos aéreas de diferentes elementos de gran importancia en la variabilidad horizontal del suelo y su tradicional uso en Edafología debido también a que facilita la cartografía de su distribución geográfica forman parte de las causas más importantes de que la Fotointerpretación Aérea (FIA) sea un instrumento metodológico que sigue manteniendo su utilidad para la realización de mapas de variabilidad del terroir o zonificación de los terroir de media y pequeña escala.

\section{Importancia de los sistemas de percepción remota como método de diferenciación}

En los mapas de escalas grandes y muy grandes la importancia de la FIA es más limitada y la utilización del método directo adquiere mayor relevancia. Este método y el tipo de muestreo en malla fija (fixed grid), en el que las observaciones se sitúan a lo largo de líneas imaginarias paralelas entre sí con igual (cuadrado) o diferente (rectángulo) separación que la existente entre las líneas, se reserva para estudios detallados y muy detallados a los que nos referimos cuando hablamos de variabilidad intraparcela. El error en los límites entre sectores (precisión) queda definido por la mitad de la distancia entre líneas y de la distancia a lo largo de ellas.

En este contexto adquiere importancia y aparece en primer plano el empleo de determinados sistemas de percepción remota como método de diferenciación principalmente por su fácil acceso, pero también porque aumenta la precisión de los límites entre sectores de diferenciación. En concreto nos referimos a los sensores que proporcionan imágenes espectrales de alta resolución.

Los sensores son aquellos elementos o equipos capaces de tomar energía (espectro electromagnético) proveniente de un objeto y convertirla en una señal que pueda ser registrada y presentada de forma adecuada para extraer información: cámaras, videos, radar, sonar... La utilización de sensores (transportados por cualquier medio: satélites, aeronaves o aviones de alta, media o baja altitud, 
tripulados o no, automóviles, ... ), y el conjunto de técnicas y procedimientos para la obtención de información sobre objetos o fenómenos (el medio natural) sin tener contacto entre el sensor y los objetos, es lo que entendemos por percepción remota (remote sensing) o teledetección.

La resolución es una medida de la habilidad que posee un sistema sensor para distinguir entre respuestas que son semejantes espectralmente o próximas espacialmente. La resolución espacial mide la menor separación angular o lineal entre dos objetos y, por lo tanto, se refiere al tamaño del objeto o característica del terreno de menor tamaño que se puede distinguir en una imagen. La resolución espectral define las longitudes de onda en las que el sensor es capaz de medir la energía reflejada: el sensor $1(0,4-$ $0,45 \mu \mathrm{m})$ tiene menor resolución espectral que el sensor $2(0,4-0,5 \mu \mathrm{m})$. La franja del espectro electromagnético más utilizada en percepción remota es la situada entre 0,3 y $15 \mu \mathrm{m}$ (ultravioleta-visible-infrarrojo). La resolución radiométrica está asociada a la sensibilidad del sensor para distinguir niveles de intensidad de la señal de retorno: una resolución de 10 bits (1024 niveles digitales) es mejor que una de 8 bits. La resolución temporal es la periodicidad con que el sensor adquiere imágenes de la misma porción de la superficie terrestre y depende de las características del transportador del sensor (altura, velocidad e inclinación) y de su diseño (ángulos de observación y de cobertura).

Los tipos de resolución citados permiten la selección de imágenes adecuadas para un objetivo determinado sea este simple o ambicioso.

En Viticultura un objetivo simple, relacionado con un elemento concreto y previamente detectado y seleccionado y sin interferencias de los demás, puede ser cartografiado utilizando este tipo de recursos. Este tipo de objetivos suele estar relacionado con la mejora anual del producto (producción anual). Por ejemplo: plagas y enfermedades, carencias en el cultivo, estrés hídrico, zonas hidromórficas o encharcables/agua, factores de calidad puntual, maduración/vendimia, clorosis, etc.

Sin embargo, para abordar un objetivo ambicioso como es la optimización y homogeneización del producto se requiere la realización del mapa de variabilidad/zonificación del terroir para lo que se ha de utilizar coordinadamente todo el conjunto de técnicas más arriba comentadas: el método directo y la teledetección. En concreto se trata de que a corto o medio plazo (futuro) mediante la gestión llevada a cabo con VP los sectores de la parcela sean lo más homogéneos posible y así también el producto lo sea.

\section{5. Índices multiespectrales}

La extracción y manejo de la información de las imágenes espectrales que se relacione directamente con una de las propiedades que se pretende valorar es una tarea que presenta ciertas dificultades. La cuestión se ha abordado desde múltiples puntos de vista y no siempre con éxito. La realización más o menos compleja de índices es la más utilizada actualmente. Los índices son combinaciones de las bandas espectrales registradas por los diferentes sensores. Se han utilizado índices que se relacionan con componentes del suelo como la textura, la humedad, la materia orgánica, los óxidos de hierro (ver p.e. PalaciosOrueta et al., 1999; Zarco et al., 2005) y teledetección térmica para evaluación del estado hídrico (Berni et al., 2009; Bellvert et al., 2014), entre otros; pero sin duda los más utilizados en Viticultura son los denominados como índices de vegetación. Existen un número importante de índices de vegetación (ver p.e. Zarco et al, 2005) y cada vez hay un número creciente de estudios que los comparan.

En Viticultura se ha utilizado frecuentemente el Normalized Difference Vegetation Index (NDVI) que se ha relacionado con el contenido en clorofila (Zarco et al., 2005), con la densidad foliar o el vigor (Hall et al., 2003, Johnson et al., 2003), con el estado hídrico de la planta (Möller et al., 2007; Acevedo-Opazo et al., 2008, Berni et al., 2009; Bellvert et al., 2013, 2014), con el índice de superficie foliar (Johnson et al., 2003, Berni et al., 2009), o con el rendimiento y la composición de la uva (Hall et al., 2011), entre otros. Además, el vigor de la planta puede ser utilizado como indicador indirecto de la producción de la vid y la calidad de la uva (Hall et al., 2002). Relacionados con el NDVI se han propuesto otros índices como el Improved Soil Adjusted Vegetation Index (MSAVI), Simple Ratio Index (SR) y el Modified.

Simple Ratio Index (MSR) que también se incluyen en la comparaciones (ver Martínez y Gómez-Miguel, 2015).

Para el cálculo del NDVI se utiliza la región espectral que corresponde al rojo ( $\mathrm{R}, \mathrm{Red})$ y al infrarrojo cercano (NIR, Near-InfraRed) de acuerdo con la relación RIRRR/RIR+RR. Existen diversas maneras de trabajar con el resultado del cálculo de NDVI. (ver p.e. Da Costa et al. 2006).

Los problemas para la discriminación de sectores relacionados con la misma respuesta a propiedades diferentes y con respuestas diferentes a idénticas condiciones, junto con la variabilidad temporal a las diferentes situaciones anuales y las diferentes situaciones de la vegetación por causa del propio ciclo o debido a condiciones climáticas, sanitarias, etc., son algunas de las causas que originan que las interpretaciones del resultado no sean fáciles y que a veces sean imposibles. La utilización de varios índices no suele ser una solución aunque si un complemento metodológico fácilmente asequible (ver Martínez y Gómez-Miguel, 2015).

En definitiva, la variabilidad sólo puede ser considerada correctamente a partir de una cartografía suficientemente detallada en la que se califiquen y cuantifiquen todas las propiedades del terroir. Por estos motivos parece lo más sensato, como se ha dicho más arriba, utilizar el conjunto de las metodologías disponibles, particularmente el método directo y la propia teledetección, de forma complementaria y aprovechando las ventajas de ambas para la elaboración adecuada de los mapas de variabilidad del terroir o mapas de zonificación del terroir.

\section{Conclusiones generales}

- Existen dos formas principales de gestión o manejo de la parcela/explotación: el Manejo Tradicional o Convencional y la Viticultura de Precisión. Aunque las exigencias tecnológicas de ambos sistemas de gestión son diferentes, tanto en uno como en otra es necesario el Mapa de Variabilidad del Terroir para la correcta definición de la diferenciación de los sectores.

- El Mapa de Variabilidad del Terroir, Mapa de los Terroir o Mapa de Zonificación del Terroir constituye la cartografía detallada de la parcela y permite conocer 
la delimitación y la caracterización de los diferentes sectores que constituyen su variabilidad.

- La Viticultura de Precisión gestiona de forma independiente y optimizada cada uno de estos sectores, permitiendo, entre otras muchas cosas, la optimización del diseño de la plantación, la selección de patrones, el laboreo, el manejo de cubiertas, la adecuación de la fertilización de fondo y mantenimiento, el ajuste de la nutrición hídrica, el diagnóstico y tratamiento de plagas y enfermedades, el seguimiento de la maduración, la elección del momento de la vendimia, etc. y todo ello de manera localizada en función las características propias de cada sector.

- El Método Directo es la forma usual de conseguir el Mapa de Variabilidad del Terroir. El Mapa realizado a partir del Método Directo delimita y caracteriza los diferentes sectores con eficacia y además aporta información sobre las propiedades del terroir, lo que permite diagnosticar las causas de la heterogeneidad y con ello la posibilidad de manejarlas adecuadamente.

- Los Mapas obtenidos de Imágenes Espectrales de Alta Resolución aportan información importante, son muy asequibles, facilitan la precisión de los límites y se obtienen más rápidamente que los realizados a partir del Método Directo. Sin embargo, este tipo de mapas separa zonas cuya diferenciación se debe a la respuesta de la planta a una determinada situación que puede estar originada por un sinfín de posibilidades y, por lo tanto varían con ellas. Estos mapas son incapaces de discriminar sectores por las propiedades de los factores del medio (clima, litología, geomorfología, suelo) que afectan a la variabilidad del terroir lo que imposibilita la actuación directa sobre ellas.

- La ejecución del Mapa realizado a partir del Método Directo se encarece a grandes escalas (estudios muy detallados) debido a la dificultad de realizar adecuadamente las delineaciones (el error con malla fija es función de la longitud de los lados). Sin embargo, la tecnología del Mapa obtenido de Imágenes Espectrales de Alta Resolución permite amortiguar este problema y disminuir el coste.

- Tanto en los mapas obtenidos por el Método Directo como los obtenidos de Imágenes Espectrales de Alta Resolución la calidad del muestreo es fundamental. Tanto la densidad de observaciones como el número de propiedades implicadas en el estudio determinan su utilidad y las directrices, a ser posible siguiendo normas internacionales, deben ser garantía de calidad.

- En definitiva, ambas metodologías, Método Directo e Imágenes Espectrales, se complementan para la realización del Mapa de Variabilidad del Terroir y, una vez que se dispone del Mapa realizado a partir del Método Directo, son posibles las interpretaciones correctas de los Mapas obtenidos de Imágenes Espectrales de Alta Resolución, ya sean anuales, estacionales, en función del estado fenológico o por cualquier otra circunstancia que pueda afectar a la variabilidad (plagas, estrés, ...).

- Por este motivo lo más sensato es, como se ha dicho, utilizar el conjunto de las metodologías disponibles, particularmente el Método Directo y la propia Teledetección, de forma complementaria para la elaboración adecuada de los Mapas de Variabilidad del Terroir o Mapas de Zonificación del Terroir.
- El Mapa de Variabilidad del Terroir así obtenido permite al viticultor abordar el objetivo más ambicioso: la optimización y homogeneización del producto. En concreto se trata de que a corto o medio plazo (futuro) y mediante la gestión llevada a cabo con la Viticultura de Precisión la actuación sobre las propiedades del terroir en los diferentes sectores de la parcela se consiga la mayor homogeneidad posible y así también el producto lo sea.

\section{Referencias}

[1] C. Acevedo-Opazo, B. Tisseyre, S. Guillaume y $\mathrm{H}$. Ojeda. The potential of high spatial resolution information to define within-vineyard zones related to vine water status. Precis.Agric. 9: 285-302 (2008)

[2] J. Bellvert, P. Zarco, E. Fereres y J. Girona. Mapping crop water stress index in a "Pinot noir" vineyard: comparing ground measurements with thermal remote sensing imagery from an unmanned aerial vehicle. Precision Agriculture Journal, 15, 4: 361-376 (2014)

[3] J.A. Berni, P.J. Zarco, L. Suárez y E. Fereres. Thermal and narrowband multispectral remote sensing for vegetation monitoring from an unmanned aerial vehicle. IEEE Trans. On Geosc. And Rem. Sens, 47, 3: $722-738$ (2009)

[4] R. Bramley y R. Hamilton. 2004. Understanding variability in winegrape production systems 1 . Within vineyard variation in yield over several vintages. Australian Journal of Grape and Wine Research, 10: 32-45

[5] R. Bramley y R. Hamilton. Terroir and precision viticulture: Are they compatible? Journal International des sciences de la vigne et du vin, 41: 1-8 (2007)

[6] S. Cook and R. Bramley. Precision agriculture opportunities, benefits and pitfalls of site-specific crop management in Australia. Aust. J. Exp. Agric. 38: 753-763 (1998)

[7] J.P. Da Costa, Ch. German, O. Lavialle, S. Homayouni y G. Grenier. Vine field monitoring using resolution remote sensing images: segmentation and characterization of rows of vines. VIth Int. Terroir Congr. Montpellier (2006)

[8] V.D. Gómez-Miguel, Terroir. In: J. Böhm (ed.) Atlas das castas da península Ibérica: história, terroir, ampelografia. pp. 104-153. Dinalivro, Lisboa, Portugal (2011)

[9] V.D. Gómez-Miguel y V. Sotés. Delimitación de zonas vitícolas de la DO Ca Rioja. Universidad Politécnica de Madrid, Madrid (1997)

[10] V.D. Gómez-Miguel. Geología, Geomorfología y Edafología. Atlas Nacional de España. Instituto Geográfico Nacional. Madrid (2005)

[11] V.D. Gómez-Miguel, M.L. González-SanJosé y V. Sotés. High resolution remote sensing a supplement to mMicro-zoning studies. IXe Congrès International des Terroirs vitivinicoles, Francia (2012)

[12] A. Hall, D. Lamb, B. Holzapfel y J. Louis. Optical remote sensing applications in viticulture - a review. Aust.J.Grape Wine Res. 8:36-47 (2002)

[13] A. Hall, D.W. Lamb, B.P. Holzapfel y J.P. Louis. Within-season temporal variation in correlations 
between vineyard canopy and winegrape composition and yield. Precis.Agric. 12:103-117 (2011)

[14] A. Hall, J. Louis y D. Lamb. Characterizing and mapping vineyard canopy using high-spatialresolution aerial multispectral images. Comput. Geosci. 29:813-822 (2003)

[15] J.R.Jensen, Introductory digital image processing: a remote sensing perspective. Prentice Hall. New Jersey 526 pp. (2004)

[16] L. Johnson, D. Roczen, S. Youkhana, R. Nemani $\mathrm{y}$ D. Bosch. Mapping vineyard leaf area with multispectral satellite imagery. Comput.Electron.Agric. 38:33-44 (2003)

[17] MAPA. Métodos oficiales de análisis de suelos y plantas. Ministerio de Agricultura, Pesca y Alimentación. Madrid (1986)

[18] A.J. Mathews y JR. Jensen. Visualizing and quantifying vineyard canopy LAI using an unmanned aerial vehicle (UAV) collected high density structure from motion point cloud. Remote Sensing, 5, 5: 2164 2183 (2013)

[19] A. Martínez, J. Cámara y V.D. Gómez-Miguel. Vegetation indices variability in vineyards of Rioja Alta (DO Ca Rioja) in different dates. X Int Cong Terroir Tokai (Hungría) (2014)

[20] A. Martínez, y V.D. Gómez-Miguel. Vegetaction index cartography as a methodology complement to the terroir zoning for its use in precision viticulture (i.p.)

[21] M.V. Möller, Alchannatis, Y. Cohen, M. Meron, J. Tsipris, A. Nahor, V. Ostrovsky, M. Sprintsin y $\mathrm{S}$. Cohen. Use of termal and visible imagery for estimating crop water status of irrigated grapevine. Jor. of Experim. Botany, 58:827-838 (2007)
[22] OIV. 2015. Preliminary Draft Resolution. VITITECVIT 14-xxx Et1. Version 01/2014 (a proposed by Bramley et al. 2005)

[23] OIV (Mod). The OIV guidelines for sampling in vineyards. CI-TECVIT 2014-03 04.WD23 (propuesta) (2015)

[24] H. Ojeda. Precision irrigation of the vineyard. CONCLIVIT. Zaragoza. España (2007)

[25] A. Palacios-Orueta, JE Pinzón, SL Ustin, y D.A. Roberts. Remote sensing of soils in the Santa Mónica Mountains. Rem Sensing of Environment, 68: 138-151 (1999)

[26] A.G. Reynolds, IV Senchuk, C. Reest y Ch. Savigny. Use of GPS and GIS for elucidation of the basis for terroir: spatial variation in an Ontario Riesling vineyard. Am. J. Enol. Vitic., 58,2:145-163 (2007)

[27] J.A. Taylor. Digital terroir and precision viticulture. PHD Thesis. MMIV. New South Wales Australia 255p. (2004)

[28] USDA. Kellogg Soil Survey Laboratory Methods Manual. USDA. Version 5.0 (2014)

[29] USDA. Soil Survey Field and Laboratory Methos Manual, USDA. Version 2 (2014)

[30] USDA. Precision Agriculture: NRCS support for emerging technologies. Agronomy Technical Note No. 1 (June 2007)

[31] E. Vaudour. Les terroir viticoles. Editions La VigneDUNOD, Paris 294 pp. (2003)

[32] P, A. Zarco, Berjón, R. López-Lozano, J. Miller, P. Martín, V. Cachorro, González M.y A. de Frutos, Assessing vineyard condition with hyperspectral indices: Leaf and canopy reflectance simulation in a row-structured discontinuous canopy. Remote Sens. Environ., 99, 3: 271-287 